\title{
Obesity and the Survival of Critically III Patients with Acute Kidney Injury: A Paradox within the Paradox?
}

\author{
Helmut Schiffl \\ Department of Internal Medicine IV, University of Munich, Munich, Germany
}

\section{Keywords}

Abdominal obesity · Epidemiology · Acute kidney injury · Intensive care medicine $\cdot$ Outcome

\begin{abstract}
The obesity epidemic is reflected by the rising number of obese patients requiring intensive care. Obesity is a recognized risk factor for the development of acute kidney injury (AKI) in critically ill patients. Both acute critical illness and AKI are associated with higher in-hospital mortality rates, and intensive care unit (ICU) patients suffering from AKI have an elevated risk of death. The relationships between obesity and mortality in critically ill paediatric and adult patients with or without AKI are less clear. Conflicting evidence exists regarding the potential impact of body mass index on the mortality of ICU patients with AKI. Some studies looking at the ICU outcomes of critically ill obese patients with AKI show reduced mortality and others show either no association or elevated mortality. Despite a high biologic plausibility of the proposed causal mechanisms, such as a greater haemodynamic stability and the protective cytokine, adipokine, and lipoprotein defence profiles associated with obesity, the inconsistency of the data suggests that the obesity
\end{abstract}

paradox is a statistical fallacy and the result of chance, bias, and residual confounding variables in retrospective cohort analyses. Further prospective randomized trials are essential to elucidate the role of obesity and the mechanisms underlying a potential survival benefit of obesity in critically ill patients with AKI.

(c) 2019 The Author(s)

Published by S. Karger AG, Basel

\section{Introduction}

Obesity is a common disorder that has serious physical, mental, social, and financial health consequences and threatens to overwhelm both industrialized and developing countries. The global epidemic of obesity affects virtually all ages and socioeconomic groups. In general, obesity is associated with greater risks for developing non-communicable diseases, disability, and premature death [1]. Given the increasing prevalence of obesity in the general population and the association of obesity with a wide spectrum of medical and surgical pathologies, it is not surprising that, compared with the general population, obese individuals require hospital admission more often [2].

\section{KARGER}

E-Mail karger@karger.com www.karger.com/kdd (c) 2019 The Author(s) Published by S. Karger AG, Basel

Karger Open access

This article is licensed under the Creative Commons AttributionNonCommercial-NoDerivatives 4.0 International License (CC BYNC-ND) (http://www.karger.com/Services/OpenAccessLicense). Usage and distribution for commercial purposes as well as any distribution of modified material requires written permission.
Prof. Dr. Helmut Schiff

Department of Internal Medicine IV, University of Munich Ziemssenstr. 1

DE-80336 Munich (Germany)

E-Mail h-schiffl@t-online.de 


\section{Obesity in the Intensive Care Unit}

Intensive care units (ICUs) have undergone a transformation in the past 3 decades, from places that used to see the occasional severely overweight patient to places that now care for multiple morbidly obese or super-obese patients (i.e., with a body mass index $[\mathrm{BMI}]>50$ ). The creeping epidemic of obesity has severely affected ICUs [3]. An audit of an Australian ICU found that approximately three-quarters of ICU patients were overweight or obese, which was approximately $12 \%$ higher than the percentage of overweight and obese individuals in the general population [4]. Reports from around the world have shown that nearly one-third of ICU patients meet the criteria for obesity, and up to $7 \%$ are morbidly obese [5-8].

Obesity in critically ill patients is typically associated with increased morbidity, a prolonged duration of mechanical ventilation, prolonged ICU stays, and frequent re-admission after hospital discharge. The results regarding the association of obesity with the risk of death are conflicting, and different studies have found that obesity is related to a reduced, an unchanged, and an elevated risk of death.

Many, mostly retrospective cohort studies, have investigated the association between BMI and in-hospital mortality and produced conflicting results regarding whether obesity modifies the risk of death in critically ill patients [9-15]. The obesity paradox is usually characterized by U- or J-shaped curves, with elevated risk levels at both the low and high ends of the BMI continuum, but the nadir or optimal BMI associated with the lowest mortality varies according to patient characteristics (age and ethnicity) and the nature of the underlying illness [16].

Meta-analyses have reported conflicting outcomes in critically ill children or adults and do not provide a clear answer to the crucial question of whether an obesity paradox exists in adult critically ill patients $[5,17,18]$. There is an unmet need for robust randomized trials comparing mortality between critically ill obese patients and those with a normal BMI. Most of the observational studies incorporated into meta-analyses have a moderate-to-high risk of bias. The percentage of missing data is high (up to $35 \%)$. Height and weight are often estimated rather than properly measured in ICU patients. Some authors define only arbitrary cut-off criteria for the overweight category (e.g., BMI >28). All studies use the BMI as a measure of obesity.

There may be several known or unknown confounders. The association of obesity and mortality risk in critically ill patients is confounded by malnutrition. In a sub- set of critically ill patients with a BMI $\geq 30.0$, mortality was greater in those with either non-specific or proteinenergy malnutrition than in well-nourished patients with comparable BMIs [5]. Moreover, in a large cohort of critically ill patients $(1,042,710 \mathrm{ICU}$ patients, 74,771 of whom received enteral nutrition with the first $48 \mathrm{~h}$ ), Harris et al. [19] observed a survival advantage with overweight and obesity. However, among patients who received early enteral nutrition, the survival disadvantage associated with a BMI $<25.0$ was minimal or unobservable. Early enteral/ parenteral nutrition prevents protein-energy malnutrition and the obesity paradox is not observed in well-nourished critically ill patients [19].

Notably, obesity (i.e., according to BMI) is not captured by prognostic indices of ICU mortality, such as the APACHE II/III and SAPS II/III scores.

\section{Critical III Obese Patients and Acute Kidney Injury}

Hospital-acquired acute kidney injury (AKI) is a multi-faceted, potentially reversible renal syndrome with an unpredictable outcome. Using current diagnostic and grading criteria (elevated serum creatinine levels and reduced urine output), it has been found that AKI occurs in $>50 \%$ of ICU patients. Clinically, AKI encompasses a broad spectrum of manifestations ranging from tubular stress, identified by the increased urinary excretion of proteins, to oligo-anuric kidney failure. Renal replacement therapy (RRT) is administered to $10-15 \%$ of ICU patients with severe AKI. The prognosis of critically ill patients with severe AKI remains poor. AKI contributes significantly to in-hospital mortality irrespective of its severity and duration, increasing the risk for de novo chronic kidney disease (CKD), the progression of CKD to endstage renal disease, and long-term cardiovascular morbidity and mortality [20].

Hospital-acquired AKI is a heterogeneous group of renal disorders with multiple aetiologies and risk factors. Obesity has been found to increase the susceptibility to AKI in critically ill patients undergoing high-risk surgery or who have sepsis/septic shock. The prevalence of AKI in the ICU is higher in obese than non-obese critically ill patients, and the prevalence of AKI in critically ill patients has been consistently shown to increase with increasing BMI. Numerous investigations have identified obesity as an independent risk factor for AKI in patients undergoing cardiac, gastrointestinal, bariatric, and trauma surgery, and in patients with acute respiratory distress syndrome (ARDS) or pancreatitis. These studies controlled 
Table 1. Evidence of a survival benefit in obese critically ill patients with AKI

\begin{tabular}{llll}
\hline First author [ref.] & Year & AKI severity & Survival paradox \\
\hline Druml [22] & 2010 & AKI-D & Yes \\
\hline Chao [23] & 2014 & AKI stages 1-3 & Yes \\
\hline Kim [24] & 2018 & AKI-D & Yes \\
\hline Kim [25] & 2017 & AKI-D & $\begin{array}{l}\text { Yes (high disease severity) } \\
\text { No (low disease severity) }\end{array}$ \\
\hline Pedersen [26] & 2017 & AKI stages 1-3 & No \\
\hline Soto [27] & 2012 & AKI stages 1-3 & No \\
\hline Danziger [28] & 2016 & AKI stages 1-3 & No \\
\hline Gameiro [29] & 2018 & AKI-stages 1-3 & No \\
\hline
\end{tabular}

AKI stages 1-3 were defined according to current classifications systems that define and grade AKI. Stage 3 represents severe AKI requiring renal replacement therapy. Disease severity was defined by the sepsis-related organ failure assessment (SOFA) score. High disease severity was defined as a SOFA score of 12 . AKI, acute kidney injury; AKI-D, acute kidney injury requiring renal replacement therapy.

for known confounders, such as co-existing chronic diabetes, hypertension, primary kidney disease, and blood product transfusion. The clinical spectrum of manifestations of obesity-associated ICU AKI encompasses all AKI grades, ranging from tubular dysfunction with mild-tomoderate increases in serum creatinine concentrations to oligo-anuric acute kidney failure requiring RRT. In obese critically ill patients, the exact prevalence of AKI, the complex pathogenetic mechanisms causing AKI, the clinical course (reversibility and development of CKD) of $\mathrm{AKI}$, and the short- and long-term outcomes are largely unknown [21].

\section{Obesity and the Risk of Mortality in Critically III Patients with AKI}

Relatively few investigations have examined the complex relationship between AKI and mortality in critically ill obese patients in detail (Table 1). The association of obesity and AKI was reviewed by our group previously. However, the number of reports has doubled during the last 3 years. To the best of my knowledge, no systemic review or meta-analysis has been published.

Conflicting evidence exists regarding the potential impact of the BMI on the outcome of critically ill patients with AKI. The available cohort analyses describe, as in other chronic or wasting disorders, the following different patterns of the relationship of obesity with AKI or mortality in obese ICU patients: (a) a non-linear (U- or J-shaped) association of obesity and mortality, (b) no association between obesity and mortality in critically ill patients with AKI, and (c) elevated mortality in critically ill obese patients with AKI.

The retrospective analyses of a large Austrian intensive care database reported by Druml et al. [22] reported, for the first time, a non-linear association between BMI and mortality in 5,232 ICU patients with severe AKI requiring RRT. These patients were divided into 1 of the following 5 BMI classes: underweight (BMI 18.5), normal weight (BMI 18.5-25), overweight (BMI 25-30), obese (BMI 3035), and morbidly obese (BMI 35). The lowest risk-adjusted hospital and ICU mortality rates were found in obese patients (odds ratio [OR] 0.81 and 0.92, respectively). Compared with obese patients, morbidly obese patients had non-significantly slightly increased rates. The authors concluded that, compared to underweight or normal-weight ICU patients with severe AKI, obese patients appeared to have improved survival [22].

Chao et al. [23] performed a retrospective analysis of a multi-centre cohort, consisting of 2,015 Chinese geriatric ICU patients who developed AKI after major surgery. Elderly AKI patients with a BMI between 21 and 31 (normal weight) had a lower mortality risk than those with a BMI $<21$ (underweight) or $\geq 31$ (obese). The U-shaped association between BMI and mortality risk found by Chao et al. [23] in geriatric ICU patients with AKI had a widened base and a shifted nadir compared to the J-shaped curve reported by Druml et al. [22].

Kim et al. [24] retrospectively examined the impact of disease severity on the association between BMI and mortality risk in 1,144 Korean ICU patients with severe AKI requiring continuous RRT. These patients are known to be highly catabolic and susceptible to the loss of energy. The study participants were categorized into the following 4 groups according the BMI classification system for Korean patients: underweight (BMI <18.5), normal weight (BMI 18.5 to $<23$ ), overweight (BMI 23 to $<25$ ), and obese $($ BMI $>25)$. Obese patients had a significantly lower risk of death (hazard ratio [HR] 0.81) than the normal-BMI group after adjusting for confounding factors. However, this association was only evident among patients with severe disease [25]. In contrast, in patients with a low disease severity, there was no survival benefit associated with a higher BMI [24]. Other retrospective analyses found no difference in the mortality risk between obese and non-obese patients with AKI. 
Pedersen et al. [26] retrospectively assessed the impact of BMI on the risk of AKI and mortality after hip fracture surgery in 56,420 elderly patients. Using Cox proportional hazard regression analyses, they showed that, compared to normal-weight patients (BMI 18.5-24.9), obese patients (BMI 30) were at an increased risk of developing AKI. However, among patients with or without post-operative AKI, neither overweight nor obesity was associated with short- or long-term (up to 1 year post-surgery) mortality.

The retrospective study of 751 ICU patients with ARDS reported by Soto et al. [27] found that obesity was associated with an increased development of AKI. While increased BMI was associated with decreased mortality, when compared to patients without AKI, those with ARDS and AKI had a significantly higher 60-day mortality, and the risk of mortality increased with increasing AKI severity.

Danziger et al. [28] retrospectively evaluated the associations of obesity, AKI, and AKI severity in 14,986 critically ill patients and investigated in-hospital and 1-year mortality. They observed that obese patients were more likely than non-obese patients to develop AKI which was associated with increased short- and long-term mortality. The associations of in-hospital and 1-year survival rates with AKI were similar across BMI categories.

Gameiro et al. [29] analysed the impact of obesity on the occurrence of AKI and in-hospital mortality in a cohort of 456 adult patients with sepsis. Compared with non-obese patients, obese patients developed AKI more frequently. However, the percentages of obese patients did not differ between AKI stages. There was no association between obesity and mortality.

The abovementioned studies had several expected limitations, namely, their retrospective design, use of body mass as an index of obesity, utilization of different definitions of BMI categories (global vs. national), use of different mortality end points, and the differences in patient characteristics such as ethnicity, existing comorbid diseases (especially metabolic syndrome), and AKI cause, severity, and treatment.

\section{Survival Paradox of Obese Critically III Patients with AKI: Myth or Fact?}

The obesity survival paradox, a medical hypothesis generated by epidemiologic investigations, describes the association of excess body weight, a complex risk factor, with premature death in the general population and, in contrast, an association of excess body weight with better outcomes in certain subpopulations of patients. A large but controversial body of evidence supporting this hypothesis has accumulated for patients with a growing number of medical disorders. The prevailing theme is that a U-shaped curve may be present when relating BMI to survival. Malnourished patients with low BMI values and those with severe (class III) obesity would be expected to have the worst outcomes. In ICU patients with AKI, this paradox is particularly surprising because, compared with non-obese patients, obese patients with acute critical illness and kidney failure have an increased risk of death. The aetiology of this presumed paradox is not clear. While of great clinical interest, the counter-intuitive findings reported in the literature may have contributed to confusion among clinicians regarding the appropriate treatment of conventional risk factors in patients with chronic disease, and physicians may be more likely to refer obese patients for intensive care treatment because they are perceived to be a group at greater risk for a poor outcome. Therefore, understanding the obesity survival paradox remains a great challenge.

\section{The Obesity Paradox in ICU Patients with AKI: A Statistical Artefact}

1 Essentially, all studies exploring the relationship between obesity and survival of critically ill patients with AKI have been retrospective analyses; they are thus limited by the inability to adjust for all confounding variables. Retrospective analyses are a tool for generating hypotheses to be tested in other settings and their results should be cautiously interpreted as an association, which does not necessarily imply causality.

2 None of the databases used was specifically designed to study the obesity paradox as a primary goal; the studies are thus limited to analysing only the available data and covariates.

3 None of the studies has data on recent weight change, i.e., unintentional weight loss in the period preceding hospital admission or during a prolonged stay in hospital.

The survival paradox of obese ICU patients with AKI can thus be criticized on the grounds of being an artefact arising from multiple sources of methodological errors (Table 2). These systematic errors or biases cannot be easily determined and addressed using statistical methods. The most common categories that can affect the validity
Schiffl 
Table 2. The obesity paradox in critically ill patients with AKI

\begin{tabular}{ll}
\hline A statistical artefact & \\
\hline $\begin{array}{l}\text { limitations of the } \\
\text { epidemiologic studies }\end{array}$ & limitations of the BMI \\
\hline $\begin{array}{l}\text { Retrospective design } \\
\text { Selection bias }\end{array}$ & $\begin{array}{l}\text { Body composition } \\
\text { Confounding }\end{array}$ \\
$\begin{array}{l}\text { Beverse causation } \\
\text { Referral/treatment bias }\end{array}$ & $\begin{array}{l}\text { Single-point (one-time) } \\
\text { measurement } \\
\end{array}$ \\
& No correction for age, sex, or race \\
& Hypervolemia \\
\hline
\end{tabular}

of the reported results include sampling selection bias, residual or unmeasured confounding, reverse causation or collider bias, and referral/treatment bias.

Medical or surgical ICU patients comprise heterogeneous, poorly defined populations. Populations of critically ill patients with or without obesity differ in the acuity of the underlying disease, in the number and severity of pre-existing comorbidities, and in the presence of organ dysfunctions that complicate the underlying illness.

Random errors, i.e., those due to sampling variability or the imprecise coding of diseases, occur in essentially all quantitative clinical studies. These inaccuracies produce a consistent false pattern of differences between observed and true outcomes. This is a risk whenever analyses are restricted to specific patient groups and can never be fully accounted for by the statistical methods used. Metaanalyses have suggested that obese patients may be at a higher risk of developing pneumonia but may have a lower risk of mortality. However, recent studies have shown that, compared with normal-weight individuals, overweight and obese people are admitted to the hospital (ICU) more often, but with less severe pneumonia (displayed by lower pneumonia index scores) and lower plasma levels of C-reactive protein [30]. The obesity paradox is frequently studied in the context of prevalent disease, a stage in the disease when confounding from illness-related weight loss and selective survival are especially prevalent. Moreover, the survival advantage in obese patients with prevalent cardiovascular disease reported in some studies disappears when investigators look at incident cardiovascular disease. Chang et al. [31] used data from the Health and Retirement Study and observed a strong and significant obesity paradox in prevalent disease models (e.g., myocardial infarction, congestive heart failure, and stroke), replicating previous findings in a population-based sample with longer-term follow-up. The HR for death was $18-36 \%$ lower for obese class I patients than for normal-weight patients. However, the authors found no evidence of a survival advantage due to obesity in patients with incident cardiovascular disease. Prevalent disease models used the concurrent weight status, and incident disease models used the pre-diagnosis weight status [31].

Given the widespread use of prevalent disease analyses in the literature, the extent of bias from disease-related confounding in individuals with prevalent disease may be unappreciated. In the ICU, the severity of illness scoring systems can be broadly divided into those that assess acute and chronic disease severity (Acute Physiology and Chronic Health Evaluation [APACHE] II and III) and those that assess the presence and severity of organ dysfunction (Sequential Organ Dysfunction Assessment [SOFA]). Kim et al. [24] evaluated whether disease activity (according to the SOFA score) can modify the relationship between BMI and the risk of mortality in critically ill patients with severe AKI. They found no survival benefit in patients with relatively less severe illness.

Finally, there is no standard obese individual. Obese patients differ in their class of obesity and in their burden of comorbid diseases. In a retrospective analysis of 351,000 patients from the American College of Surgeons National Surgical Quality Improvement database, Glance et al. [32] identified a subpopulation of obese patients with metabolic syndrome who had a dramatically elevated risk of post-operative complications. Specifically, compared with other patients, obese patients with metabolic syndrome experienced a 2- to 3-fold higher risk of cardiovascular events, a 1.5 - to 2.5 -fold higher risk of pulmonary complications, a 2-fold higher risk for neurologic catastrophes, a 3- to 7-fold higher risk of AKI, and a 2 -fold higher risk of death.

BMI has traditionally been the measure of obesity in all studies examining the outcomes for ICU patients with AKI and in nearly all studies dealing with the survival of critically ill patients. Describing obesity according to BMI can result in an inaccurate assessment of adiposity because the numerator in the calculation does not distinguish lean muscle from fat mass. The clinical utility of BMI could thus be questioned because it does not accurately reflect visceral fat accumulation, the likely culprit leading to most of the metabolic and clinical consequences of obesity. Thus, a person with central obesity (with excessive visceral fat mass) can have a normal BMI and yet be at a high risk due to the consequences of obesity. BMI does not take into consideration sex differences in the distribution of fat or age-related decline in muscle 
mass. There is growing recognition of a metabolically healthy obesity state, in which some individuals are free from the metabolic complications of obesity, most likely due to reduced amounts of visceral fat and preserved insulin sensitivity. Other relatively simple methods of measuring body fat include the waist-to-hip ratio, waist circumference, and skin-fold thickness. More sensitive but costly measures include bio-electrical impedance analysis, computed tomography, and magnetic resonance imaging. The results of studies using alternative measures of obesity in addition to BMI, such as the waist circumference or the waist-to-hip ratio, have rendered the existence of the obesity paradox questionable [33, 34].

Inaccuracies in the measurement of single-point BMI during acute illness, e.g., due to hypervolemia resulting from AKI or tissue oedema resulting from fluid resuscitation in septic patients, may have created a misclassification of normal or overweight patients.

Measurements of the weight and height of ICU patients are actually not always performed. Patients are frequently admitted in an emergency, are not ambulant, or may unable to communicate; these factors, alone or in combination, can make accurate measurement of height and weight difficult. Visual estimations of the weight and height of ICU patients by the medical and nursing staff are frequently inaccurate, vary according to the ability of the observer, and result in frequent and clinically relevant overestimation of low-weight patients and the underestimation of obese patients. Errors in the estimation of weight and height could compromise the application of BMI for use in research $[35,36]$.

Alternatively, treatment and referral bias could account for the survival paradox in critically ill obese patients with AKI. Clinicians tend to consider obese patients as having a high risk of poor outcomes. This view might result in earlier admission of not so critically ill obese patients with more easily treatable infections to the ICU for monitoring purposes and also early or increased use of prophylactic measures (nutrition).

In addition, obesity highlights potential hazards and may persuade physicians to more stringently adhere to guideline-based management, such as high-calorie nutrition therapy, early mobilization, cautious pressure ulcer prevention, strict glycaemic control, and more attention to mechanical ventilation parameters [37]. Arabi et al. [38] postulated that obese and non-obese patients with sepsis are managed differently during resuscitation and antibiotic therapy. Because these therapeutic interventions did not take into consideration the large variations in BMI among the study participants, the patients with class III obesity received half the volume of crystalloid and colloid resuscitation fluids than the underweight patients in the first $6 \mathrm{~h}$. Antimicrobial doses per kilogram were also different across BMI groups. Crude analyses showed that obese and very obese patients had a lower hospital mortality rate than normal-weight patients. After adjusting for baseline characteristics and sepsis interventions, the association between BMI and survival became nonsignificant. The authors concluded that the obesity paradox may relate to differences in patient characteristics and variations in sepsis intervention.

It may be the case that low BMI does not cause death (thereby apparently making obesity protective), but rather that imminent death may be the cause of the low BMI. Indeed, unintentional weight loss is an extremely significant predictor of mortality. Sick individuals (such as critically ill patients with AKI) often undergo relevant weight loss before death (due to uraemia, hypercatabolism, inadequate nutrition, the loss of nutrients via renal replacement correction of AKI-induced, or resuscitation-related hypervolemia by dialysis), and classifying these individuals as lean greatly inflates the mortality rate of the normal-weight and underweight BMI categories and lowers the risk in the higher BMI category. Indeed, studies that employed strategies to reduce reverse causation, such as excluding sick individuals at the baseline and introducing a time lag to exclude deaths at the beginning of follow-up, have yielded estimates of an increased risk of death for patients with a $\mathrm{BMI}>25$ [39].

\section{The Obesity Paradox of Critically III Patients with AKI Is True}

The clinical data has repeatedly shown improved survival of obese patients with chronic disorders or acute illness. The underlying pathophysiological mechanisms have not been investigated in ICU patients with AKI and remain largely unclear in critically ill patients (Fig. 1).

One often-discussed argument for a survival advantage of obesity is the elevated metabolic reserve during cachectic states. Unintentional weight loss is a frequent finding associated with acute, life-threatening disease, and may lead to malnutrition which has a negative impact on immune function. The greater muscle mass and larger amount of subcutaneous fat in obese patients may make them more resistant to the catabolic progression of wasting diseases. Aquilani et al. [40] found a balanced muscle
18

Kidney Dis 2020;6:13-21

DOI: $10.1159 / 000502209$
Schiffl 
Fig. 1. Several pathophysiological mechanisms haven been postulated to explain the obesity paradox in critically ill patients. First, obesity may be associated with higher metabolic reserves which are beneficial in acute illnesses with severe catabolism. Second, heightened activity of the renin-angiotensin-aldosterone system (RAAS) or sympathetic nervous system may confer haemodynamic advantages in sepsis. Third, obesity may result in a form of inflammatory preconditioning. Fourth, the augmented secretion of lipoproteins and anti-inflammatory cytokines by adipose tissue may mitigate the adverse sequelae of bacterial infection.

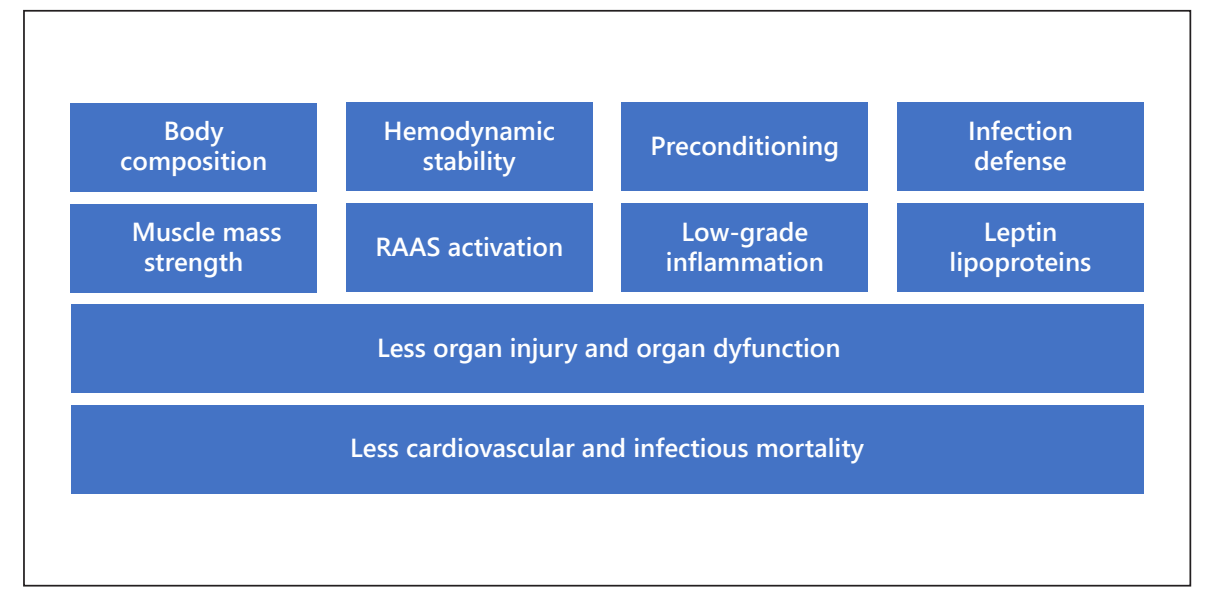

protein catabolism only in obese patients, but not in normal-weight patients with heart failure, and it is well known that unbalanced protein catabolism is associated with reduced immunologic capacity.

Preconditioning via a chronic pro-inflammatory state may create a protective environment, limiting the detrimental effects of a more aggressive second hit, such as organ injury or sepsis. Indeed, obesity itself increases the plasma and adipose tissue levels of inflammatory cytokines and induces low-grade inflammation, generating a process (organ crosstalk) that subsequently protects organs against insults [5, 41-43].

The secretion of leptin (an adipokine) by the adipose cells of white adipose tissue is mediated in response to sepsis or stress. Plasma leptin levels are elevated in obese patients and even more elevated in patients with sepsis. Preventive administration of leptin to rats exposed to endotoxaemia has been shown to protect against the development of multiple-organ dysfunction syndrome and reduce the risk of death. Leptin may augment the immune response and improve bacterial clearance in experimental animals. Circulating leptin levels are increased in individuals with sepsis. Hyperleptinemia independently predicts the development of sepsis and its outcome [44]. When compared with critically ill patients with sepsis who died, those who survived had 3 -fold higher plasma concentrations of leptin [45]. Obesity is associated with higher circulating levels of highdensity lipoprotein that binds bacterial lipopolysaccharides (endotoxins) [46]). Increased activation of the renin angiotensin system by obesity may confer haemodynamic advantages in patients with sepsis and may be associated with less hypotension-mediated ischaemia of vital organs [47].

Obesity Paradox and ICU-AKI

\section{Conclusions}

Obesity is an independent risk factor for AKI in critically ill patients. Whether the survival benefit of obesity in critically ill patients with AKI (a paradox within a paradox) is due to plausible biological reactions or to methodological errors remains controversial, especially given the lack of clearly defined pathophysiologic mechanisms. Currently, number, size, and quality of published analyses are low. Admittedly, adiposity is not always unhealthy, depending on the amount of fat accumulated, the regional fat distribution, and the metabolic activity of the adipose tissue. However, there is no definitive clinical evidence of a survival benefit of obesity in critically ill patients with or without AKI.

The conflicting evidence regarding the potential impact of obesity on mortality in critically ill patients does not offer support for re-evaluating clinical and public health guidelines in pursuit of the potential obesity paradox. No one should suggest that gaining body weight improves the odds of a healthy outcome for any illness or misinform patients who are at a high risk of cardiovascular disease. Ultimately, any positive causal connection between obesity and survival in a specific scenario is outweighed by the volume of data linking obesity with a great number of severe illnesses. Any residual uncertainty should not mean that physicians overlook the clear risklowering effects of weight reduction in obese individuals who are at a high risk of developing metabolic, renal, or cardiovascular disorders.

Further studies are warranted to explain and interpret this paradoxical relationship and define the optimal weight range for the critically ill patient population. Ideally, randomized trials should take into consideration the 
limitations and biases of reported studies and assess the effects of unintentional weight loss on primary (i.e., mortality) and secondary (i.e., morbidity) outcomes in critically ill obese patients with or without AKI.

\section{Disclosure Statement}

The author has no conflicts of interest to declare.

\section{References}

1 Vos T, Barber RM, Bell B, Bertozzi-Villa A, Biryukov S, Bolliger I, et al.; Global Burden of Disease Study 2013 Collaborators. Global, regional, and national incidence, prevalence, and years lived with disability for 301 acute and chronic diseases and injuries in 188 countries, 1990-2013: a systematic analysis for the Global Burden of Disease Study 2013. Lancet. 2015 Aug;386(9995):743-800.

2 Fusco KL, Robertson HC, Galindo H, Hakendorf $\mathrm{PH}$, Thompson $\mathrm{CH}$. Clinical outcomes for the obese hospital inpatient: an observational study. SAGE Open Med. 2017 Mar;5: 2050312117700065.

3 Schetz M, De Jong A, Deane AM, Druml W, Hemelaar P, Pelosi P, et al. Obesity in the critically ill: a narrative review. Intensive Care Med. 2019 Jun;45(6):757-69.

4 Dennis DM, Trevenen M. Prevalence of obesity in an intensive care unit patient population. Intensive Crit Care Nurs. 2016 Aug;35: 52-6.

5 Robinson MK, Mogensen KM, Casey JD, McKane CK, Moromizato T, Rawn JD, et al. The relationship among obesity, nutritional status, and mortality in the critically ill. Crit Care Med. 2015 Jan;43(1):87-100.

6 Sakr Y, Madl C, Filipescu D, Moreno R, Groeneveld J, Artigas A, et al. Obesity is associated with increased morbidity but not mortality in critically ill patients. Intensive Care Med. 2008 Nov;34(11):1999-2009.

7 Shashaty MG, Stapleton RD. Physiological and management implications of obesity in critical illness. Ann Am Thorac Soc. 2014 Oct; 11(8):1286-97.

8 Wacharasint $P$, Fuengfoo P, Rangsin R, Morakul S, Chittawattanarat K, Chaiwat O. Prevalence and Impact of Overweight and Obesity in Critically Ill Surgical Patients: Analysis of THAI-SICU Study. J Med Assoc Thai. 2016 Sep;99 Suppl 6:S55-S62.

9 Yokoyama H, Higuma T, Endo T, Nishizaki F, Hanada K, Yokota T, et al. Prognostic impact of body mass index and culprit lesion calcification in patients with acute myocardial infarction. Heart Vessels. 2019. Epub ahead of print.

10 Samanta R, Narayan A, Kovoor P, Thiagalingam A: Influence of BMI on Short and LongTerm Outcomes in Patients with STEMI and LV Dysfunction. Heart Lung Circ. 2019. Epub ahead of print.
11 Pepper DJ, Demirkale CY, Sun J, Rhee C, Fram D, Eichacker P, et al. Does Obesity Protect Against Death in Sepsis? A Retrospective Cohort Study of 55,038 Adult Patients. Crit Care Med. 2019 May;47(5):643-50.

12 Modig K, Erdefelt A, Mellner C, Cederholm T, Talbäck M, Hedström M. "Obesity Paradox" Holds True for Patients with Hip Fracture: A Registry-Based Cohort Study. J Bone Joint Surg Am. 2019 May;101(10):888-95.

13 Lee DH, Giovannucci EL. The Obesity Paradox in Cancer: Epidemiologic Insights and Perspectives. Curr Nutr Rep. 2019 Sep;8(3): $175-81$.

14 Caan BJ, Cespedes Feliciano EM, Kroenke $\mathrm{CH}$. The Importance of Body Composition in Explaining the Overweight Paradox in Cancer-Counterpoint. Cancer Res. 2018 Apr; 78(8):1906-12.

15 Janice P, Shaffer R, Sinno Z, Tyler M, Ghosh J. The obesity paradox in ICU patients. Conf Proc IEEE Eng Med Biol Soc. 2017 Jul;2017: 3360-4.

16 Dixon JB, Lambert GW. The obesity paradox - a reality that requires explanation and clinical interpretation. Atherosclerosis. 2013 Jan;226(1):47-8.

17 Pepper DJ, Sun J, Welsh J, Cui X, Suffredini $\mathrm{AF}$, Eichacker PQ. Increased body mass index and adjusted mortality in ICU patients with sepsis or septic shock: a systematic review and meta-analysis. Crit Care. 2016 Jun;20(1):181.

18 Zhao Y, Li Z, Yang T, Wang M, Xi X. Is body mass index associated with outcomes of mechanically ventilated adult patients in intensive critical units? A systematic review and meta-analysis. PLoS One. 2018 Jun; 13(6):e0198669.

19 Harris K, Zhou J, Liu X, Hassan E, Badawi O. The Obesity Paradox Is Not Observed in Critically Ill Patients on Early Enteral Nutrition. Crit Care Med. 2017 May;45(5):828-34.

20 Schiffl H, Lang SM, Fischer R. Long-term outcomes of survivors of ICU acute kidney injury requiring renal replacement therapy: a 10 year prospective cohort study. Clin Kidney J. 2012 Aug;5(4):297-302.

21 Schiffl H, Lang SM. Obesity, acute kidney injury and outcome of critical illness. Int Urol Nephrol. 2017 Mar;49(3):461-6.

22 Druml W, Metnitz B, Schaden E, Bauer P, Metnitz PG. Impact of body mass on incidence and prognosis of acute kidney injury requiring renal replacement therapy. Intensive Care Med. 2010 Jul;36(7):1221-8.
23 Chao CT, Wu VC, Tsai HB, Wu CH, Lin YF, Wu KD, et al.; NSARF Group. Impact of body mass on outcomes of geriatric postoperative acute kidney injury patients. Shock. 2014 May;41(5):400-5.

24 Kim H, Kim H, Lee M, Cha MU, Nam KH, An SY, et al. The impact of disease severity on paradoxical association between body mass index and mortality in patients with acute kidney injury undergoing continuous renal replacement therapy. BMC Nephrol. 2018 Feb;19(1):32.

25 Kim H, Kim J, Seo C, Lee M, Cha MU, Jung $\mathrm{SY}$, et al. Body mass index is inversely associated with mortality in patients with acute kidney injury undergoing continuous renal replacement therapy. Kidney Res Clin Pract. 2017 Mar;36(1):39-47.

26 Pedersen AB, Gammelager H, Kahlert J, Sorensen HT, Christiansen CF. Impact of body mass index on risk of acute kidney injury and mortality in elderly patients undergoing hip fracture surgery. Osteoporos Int. 2017 Mar; 28(3):1087-97.

27 Soto GJ, Frank AJ, Christiani DC, Gong MN . Body mass index and acute kidney injury in the acute respiratory distress syndrome. Crit Care Med. 2012 Sep;40(9):2601-8.

28 Danziger J, Chen KP, Lee J, Feng M, Mark RG, Celi LA, et al. Obesity, Acute Kidney Injury, and Mortality in Critical Illness. Crit Care Med. 2016 Feb;44(2):328-34.

29 Gameiro J, Gonçalves M, Pereira M, Rodrigues N, Godinho I, Neves M, et al. Obesity, acute kidney injury and mortality in patients with sepsis: a cohort analysis. Ren Fail. 2018 Nov;40(1):120-6.

30 Nie W, Zhang Y, Jee SH, Jung KJ, Li B, Xiu Q. Obesity survival paradox in pneumonia: a meta-analysis. BMC Med. 2014 Apr;12(1):61.

31 Chang VW, Langa KM, Weir D, Iwashyna TJ. The obesity paradox and incident cardiovascular disease: A population-based study. PLoS One. 2017 Dec;12(12):e0188636.

32 Glance LG, Wissler R, Mukamel DB, Li Y, Diachun CA, Salloum R, et al. Perioperative outcomes among patients with the modified metabolic syndrome who are undergoing noncardiac surgery. Anesthesiology. 2010 Oct;113(4):859-72.

33 Chrysant SG, Chrysant GS. New insights into the true nature of the obesity paradox and the lower cardiovascular risk. J Am Soc Hypertens. 2013 Jan-Feb;7(1):85-94. 
34 Streng KW, Voors AA, Hillege HL, Anker SD, Cleland JG, Dickstein K, et al. Waist-to-hip ratio and mortality in heart failure. Eur $\mathrm{J}$ Heart Fail. 2018 Sep;20(9):1269-77.

35 Bloomfield R, Steel E, MacLennan G, Noble DW. Accuracy of weight and height estimation in an intensive care unit: implications for clinical practice and research. Crit Care Med. 2006 Aug;34(8):2153-7.

36 Coe TR, Halkes M, Houghton K, Jefferson D. The accuracy of visual estimation of weight and height in pre-operative supine patients. Anaesthesia. 1999 Jun;54(6):582-6.

37 Pompilio CE, Pelosi P, Castro MG. The Bariatric Patient in the Intensive Care Unit: pitfalls and Management. Curr Atheroscler Rep. 2016 Sep;18(9):55.

38 Arabi YM, Dara SI, Tamim HM, Rishu AH, Bouchama A, Khedr MK, et al.; Cooperative Antimicrobial Therapy of Septic Shock (CATSS) Database Research Group. Clinical characteristics, sepsis interventions and out- comes in the obese patients with septic shock: an international multicenter cohort study. Crit Care. 2013 Apr;17(2):R72.

39 Whitlock G, Lewington S, Sherliker P, Clarke $\mathrm{R}$, Emberson J, Halsey J, et al.; Prospective Studies Collaboration. Body-mass index and cause-specific mortality in 900000 adults: collaborative analyses of 57 prospective studies. Lancet. 2009 Mar;373(9669):1083-96.

40 Aquilani R, La Rovere MT, Febo O, Boschi F, Iadarola P, Corbellini D, et al. Preserved muscle protein metabolism in obese patients with chronic heart failure. Int J Cardiol. 2012 Oct; 160(2):102-8.

41 Hafner S, Hillenbrand A, Knippschild U, Radermacher P. The obesity paradox and acute kidney injury: beneficial effects of hyper-inflammation? Crit Care. 2013 Dec; 17(6): 1023

42 Fernandez-Bustamante A, Repine JE. Adipose-Lung Cell Crosstalk in the Obesity-ARDS Paradox. J Pulm Respir Med. 2013;3:1-7.
43 De Schutter A, Kachur S, Lavie CJ, Boddepalli RS, Patel DA, Milani RV. The impact of inflammation on the obesity paradox in coronary heart disease. Int J Obes. 2016 Nov; 40(11):1730-5.

44 Jacobsson S, Larsson P, Johansson G, Norberg M, Wadell G, Hallmans G, et al. Leptin independently predicts development of sepsis and its outcome. J Inflamm (Lond). 2017 Sep; 14(1): 19 .

45 Bornstein SR, Licinio J, Tauchnitz R, Engelmann L, Negrão AB, Gold P, et al. Plasma leptin levels are increased in survivors of acute sepsis: associated loss of diurnal rhythm, in cortisol and leptin secretion. J Clin Endocrinol Metab. 1998 Jan;83(1):280-3.

$46 \mathrm{Ng}$ PY, Eikermann M. The obesity conundrum in sepsis. BMC Anesthesiol. 2017 Oct; 17(1):147.

47 Kershaw EE, Flier JS. Adipose tissue as an endocrine organ. J Clin Endocrinol Metab. 2004 Jun;89(6):2548-56. 